\title{
Editorial: CFD Applications in Nuclear Engineering
}

\author{
Wenxi Tian ${ }^{1 *}$, Mingjun Wang ${ }^{1}$, Victor Petrov ${ }^{2}$, Nejdet Erkan ${ }^{3}$ and Yixiang Liao ${ }^{4}$ \\ ${ }^{1}$ Xi'an Jiaotong University, Xi'an, China, ${ }^{2}$ University of Michigan, Ann Arbor, MI, United States, ${ }^{3}$ The University of Tokyo, Bunkyō, \\ Japan, ${ }^{4}$ Helmholtz-Zentrum Dresden-Rossendorf, Helmholtz-Gemeinschaft Deutscher Forschungszentren (HZ), Dresden, \\ Germany
}

Keywords: CFD, nuclear reactor, two-phase flow, thermal-hydraulics and safety analyses, Best practice guidline

Editorial on the Research Topic

\section{CFD Applications in Nuclear Engineering}

Computational fluid dynamics (CFD) and computational multiphase fluid dynamics (CMFD) methods have attracted great attentions in predicting single-phase and multiphase flows under steady-state or transient conditions in the field of nuclear reactor engineering. The CFD research circle is rapidly expanding, and the CFD topic has been covered in many international conferences on nuclear engineering, such as ICONE, NURETH, NUTHOS, and CFD4NRS, which greatly extends the forum to exchange information in the application of CFD codes to nuclear reactor safety issues.

Currently, more and more scholars are devoting their efforts to CFD study in the nuclear

OPEN ACCESS

Edited by:

Muhammad Zubair,

University of Sharjah, United Arab Emirates

Reviewed by:

Ivo Kljenak,

Institut Jožef Stefan (IJS), Slovenia

*Correspondence:

Wenxi Tian

wxtian@mail.xjtu.edu.cn

Specialty section:

This article was submitted to Nuclear Energy,

a section of the journal Frontiers in Energy Research

Received: 17 November 2020 Accepted: 04 January 2021 Published: 09 February 2021

Citation:

Tian W, Wang M, Petrov V, Erkan N and Liao Y (2021) Editorial: CFD Applications in Nuclear Engineering.

Front. Energy Res. 9:630305. doi: 10.3389/fenrg.2021.630305 engineering community, and a series of valuable research results have emerged in recent years. Therefore, this research topic was proposed, and the issue was organized by Tian from Xi'an Jiaotong University, Petrov from University of Michigan, Erkan from the University of Tokyo, Liao from Helmholtz-Zentrum Dresden-Rossendorf, and Wang from Xi'an Jiaotong University, aiming to share the most advanced progress and innovations related to CFD study in nuclear engineering around the world.

In this topic, the CFD simulation in rod bundles is carried out, and the simulation results are validated based on the LDA measurement in a $5 \times 5$ rod bundle installed with two split-mixing-vane grids (Xiong et al.). The models of internal heating and natural convention buoyancy, as well as the models of WMLES turbulence and phase changing, were applied in the open-source CFD software OpenFOAM to perform numerical simulations of the COPRA single-layer molten pool experiment (Xi et al.). Three-dimensional computational fluid dynamic (CFD) simulations were performed to study the long-term heat removal mechanisms in the General Atomics' Modular High Temperature Gas-cooled Reactor (MHTGR) design during a P-LOFC accident (Wang et al.). The transient hydraulic characteristics of multistage centrifugal pump during start-up process were also studied using the CFD method (Long et al.).

In terms of two-phase flow simulation using the CFD method, the capabilities and advantages provided by a model that includes an elliptic-blending Reynolds stress turbulence closure (EB-RSM), allowing fine resolution of the velocity field in the near-wall region, are tested over a large database (Colombo and Fairweather). Ling et al. (2020) present a numerical simulation of subcooled flow boiling at a high-pressure condition. An interface tracking method, VOSET, was used to handle the moving interface, and conjugate heat transfer between the wall and the fluid was included in the numerical model. A comparison of the CFD simulation results with the high-resolution experimental data from a helical coil experimental setup operated with a mixture of water and air is discussed, with special emphasis on two-phase pressure drops and void fraction distributions (Che et al.). Zeng et al. 
studied the detailed helium bubble rising behavior in the crosstype channel using CFD software ANSYS Fluent.

With the rapid development of the GEN-IV reactors, the application of CFD in liquid metal flow and heat transfer is also widely accepted. Chai et al. performed the wall-resolved large-eddy simulation (LES) to study the flow and heat transfer properties in a turbulent channel at low Prandtl number. The numerical study on the 19-pin wire-wrapped assembly cooled by lead-bismuth eutectic in liquid metal cooled reactor was also carried out using the CFD method (Li et al.).

Park et al. summarizes the recent activities in the development of SOPHIA code using smoothed particle hydrodynamics (SPH), which is effective to solve the nuclear safety issues encountered in natural disasters and severe accidents accompanied by highly nonlinear deformations. Numerical simulation and validation of aerosol particle removal by water spray droplets with OpenFOAM during the Fukushima daiichi fuel debris retrieval was performed by considering the collection mechanisms of inertial impaction, interception, and Brownian diffusion (Liang et al.).

Finally, 12 articles in total from the United States, Europe, Japan, Korea, and China were collected, to show the recent progress of CFD study in nuclear engineering around the world. This research topic covers across both light water reactors and liquid metal cooled reactors and is definitely a pioneer in this field. It provides valuable references, guidelines, and is leading a fast-forwarding progress for the application of CFD in the nuclear reactor thermal hydraulic analysis. Anyway, we have to confess that the CFD is still developing, and more efforts are required to make it play a more significant role in the nuclear reactor design and safety analysis. We are planning to initiate a new special issue on the "CFD in Numerical Nuclear Reactor" in future. Please feel free to contact us if you have any questions or suggestions.

\section{AUTHOR CONTRIBUTIONS}

WT, leading guest editor; MW, guest editor; VP, guest editor; NE, guest editor; and YL, guest editor

Conflict of Interest: The authors declare that the research was conducted in the absence of any commercial or financial relationships that could be construed as a potential conflict of interest.

Copyright $\odot 2021$ Tian, Wang, Petrov, Erkan and Liao. This is an open-access article distributed under the terms of the Creative Commons Attribution License (CC BY). The use, distribution or reproduction in other forums is permitted, provided the original author(s) and the copyright owner(s) are credited and that the original publication in this journal is cited, in accordance with accepted academic practice. No use, distribution or reproduction is permitted which does not comply with these terms. 